PROCEEDINGS OF THE AMERICAN MATHEMATICAL SOCIETY

Volume 135, Number 8, August 2007, Pages 2453-2459

S 0002-9939(07)09008-9

Article electronically published on March 29, 2007

\title{
ASYMPTOTICS OF EIGENVALUE CLUSTERS FOR SCHRÖDINGER OPERATORS ON THE SIERPIŃSKI GASKET
}

\author{
KASSO A. OKOUDJOU AND ROBERT S. STRICHARTZ
}

(Communicated by Michael T. Lacey)

\begin{abstract}
In this note we investigate the asymptotic behavior of spectra of Schrödinger operators with continuous potential on the Sierpiński gasket $S G$. In particular, using the existence of localized eigenfunctions for the Laplacian on $S G$ we show that the eigenvalues of the Schrödinger operator break into clusters around certain eigenvalues of the Laplacian. Moreover, we prove that the characteristic measure of these clusters converges to a measure. Results similar to ours were first observed by A. Weinstein and V. Guillemin for Schrödinger operators on compact Riemannian manifolds.
\end{abstract}

\section{InTRODUCTION AND MOTIVATIONS}

The construction by Kigami [9] of a Laplacian on the post critically finite (pcf) fractals spurs new research on analysis on fractals. In particular, numbers of results in this area are reminiscent of results from analysis on manifolds, while other results are only true in this fractal setting. We refer to [1, 10, 13, 14] and the references therein for more background on analysis on fractals. In this note we consider the typical example of a pcf fractal, namely the Sierpiński gasket denoted by $K=S G$. This is the attractor of the iterated function systems (ifs) consisting of the functions $F_{1}, F_{2}$ and $F_{3}$ defined on $\mathbb{R}^{2}$ respectively by $F_{1}(x)=\frac{1}{2} x, F_{2}(x)=\frac{1}{2} x+\left(\frac{1}{2}, 0\right)$ and $F_{3}(x)=\frac{1}{2} x+\left(\frac{1}{4}, \frac{\sqrt{3}}{4}\right)$. In other words, the Sierpiński gasket is the unique nonempty compact subset of $\mathbb{R}^{2}$ such that $K=\bigcup_{i=1}^{3} F_{i} K$. Alternatively, $S G$ can be defined as a limit of graphs. For a word $\omega=\left(\omega_{1}, \omega_{2}, \ldots, \omega_{m}\right)$ of length $m$, the set $F_{\omega} K=F_{\omega_{1}} \circ \cdots \circ F_{\omega_{m-1}} \circ F_{\omega_{m}} K$ with $\omega_{i} \in\{1,2,3\}$ is called an $m$-cell. Let $V_{0}=\left\{(0,0),(1,0),\left(\frac{1}{2}, \frac{\sqrt{3}}{2}\right)\right\}$, i.e., $V_{0}$ is the set of fixed points of the contractions $F_{i}$. Let $V_{n}=\bigcup_{i=1}^{3} F_{i} V_{n-1}, n \geq 1$, and define a sequence of graphs $\Gamma_{m}$ with vertices in $V_{m}$ and edge relation $x \sim_{m} y$ given inductively by: $\Gamma_{0}$ is the complete graph with vertices in $V_{0}$, and $x \sim_{m} y$ if and only if $x$ and $y$ belong to the same $m$-cell $F_{\omega} K$. Notice also that $K$ is the closure of $V_{*}$ in the Euclidean metric, where $V_{*}=\bigcup_{n=0}^{\infty} V_{n}$. In all that follows, we assume that $K$ is equipped with the probability measure $\mu$ that assigns the measure $3^{-m}$ to each $m$ cell.

Received by the editors January 9, 2006.

2000 Mathematics Subject Classification. Primary 35P20, 28A80; Secondary 42C99, 81Q10.

Key words and phrases. Analysis on fractals, Schrödinger operators, Sierpiński gasket.

The research of the second author was supported in part by the National Science Foundation, grant DMS-0140194. 
A Laplacian on $S G$ can be defined as a renormalized limit of a graph Laplacian in the following way. First, a graph Laplacian $\Delta_{m}$ is defined on the graph $\Gamma_{m}$ by

$$
\Delta_{m} f(x)=\sum_{y \sim_{m} x} f(y)-4 f(x)
$$

for $x \in V_{m} \backslash V_{0}$. A Laplacian on $S G$ can now be defined by

$$
\Delta=\frac{3}{2} \lim _{m \rightarrow \infty} 5^{m} \Delta_{m}
$$

Using the spectral decimation method of Rammal and Toulouse [11, Fukushima and Shima gave a complete characterization of the spectrum of the Laplacian on $S G$ [5. Further description of this spectrum has been investigated in [4, 15. The tenet of the spectral decimation method is based on the fact that the eigenvalues and the eigenfunctions of $\Delta$ on $S G$ are completely determined by the eigenvalues and eigenfunctions of the graph Laplacians $\Delta_{m}$. More specifically, for every Dirichlet eigenvalue $\lambda$ of $\Delta$ on $S G$, there exists an integer $m \geq 1$ ("the generation of birth"), such that every $\lambda$-eigenfunction $f$, when restricted to $V_{m}$, is an eigenfunction of $\Delta_{m}$ with eigenvalue 2,5 or 6 . Additionally, 2 occurs only for $m=1$, while 6 occurs only for $m>1$. Moreover, the restriction of $f$ to $V_{k}$ for $k>m$ is an eigenfunction of $\Delta_{k}$ with eigenvalue $\gamma_{k}$ given by $\gamma_{k-1}=\gamma_{k}\left(5-\gamma_{k}\right)$. Furthermore, the eigenvalue $\lambda$ is related to the graph eigenvalues by

$$
\lambda=\frac{3}{2} \lim _{k \rightarrow \infty} 5^{k} \gamma_{k} .
$$

The eigenvalues obtained from the graph eigenvalue 2, i.e., the generation of birth is $m=1$, are called the 2 -series eigenvalues and all have multiplicity 1 . However, the 5 -series and the 6-series eigenvalues have very high multiplicities. A similar description for the Neumann eigenvalues exists and can be found in 14] and the references therein. A unique feature of the spectrum of the Laplacian on $S G$ (and other pcf fractals) is the existence of localized eigenfunctions [2, 5. These are eigenfunctions which satisfy both Dirichlet and Neumann boundary conditions. In particular, every 6-series eigenspace starting with a large $m$ is spanned by localized eigenfunctions, and a similar statement is true for most of the 5-series eigenfunctions. Moreover, it is known that the proportion of localized eigenvalues of $\Delta$ is close to 1 [14. Another feature of the Laplacian on $S G$, which we heavily use below, is the existence of gaps in its spectrum.

Our goal in this note is to study the asymptotics of the spectrum of the Schrödinger operator $H=-\Delta+\chi$ on $S G$, where the potential $\chi$ is a real-valued continuous function defined on $S G$. However, we first reduce the problem to the case where $\chi$ is a step function on $S G$. In this case the existence of localized eigenfunctions for $\Delta$ implies that the spectrum of the Schrödinger operator breaks into clusters whose asymptotic distribution may be described precisely. These clusters appear very naturally due to the existence of gaps in the spectrum of $\Delta$. By then approximating every continuous function on $S G$ by such step functions we establish the same result for a general Schrödinger operator with continuous potential. Notice that some experimental results pertaining to the Schrödinger equation on the Sierpiński gasket were first introduced in [3. Finally, we wish to point out that a result similar to ours was obtained by Weinstein [16] for the Schrödinger operator corresponding to the Laplace-Beltrami operator on a compact Riemannian manifold, with a smooth potential; see also 6, 7, 8. In particular, the eigenvalues of the Laplace-Beltrami operator $-\Delta$ on the unit $n$-sphere $S^{n}$ are of the form $k(k+n-1)$, for $k=0,1,2, \ldots$, 
and where the multiplicity of each eigenvalue grows as a polynomial of order $n-1$ in $k$. It was shown in [16] that the eigenvalues of the Schrödinger operator on $S^{n}$ break into clusters whose distribution converges to a certain measure when $k$ tends to infinity. However, the techniques used in the above paper are completely different from ours and rely on the well established theory of pseudodifferential operators on Riemannian manifolds, which as yet has no analog in this fractal setting.

We begin by proving the following lemma, which uses a mini-max argument (12]) to compare the eigenvalues of two Schrödinger operators with different potential. More precisely we have the following result.

Lemma 1. For $i=1,2$, let $\chi_{i}$ be real-valued bounded measurable functions on $S G$. Let $H_{i}=-\Delta+\chi_{i}$ denote the corresponding Schrödinger operators. For $n \geq 1$, the nth eigenvalues $\nu_{n}^{i}$ of $H_{i}, i=1,2$, satisfy the following inequality:

$$
\left|\nu_{n}^{1}-\nu_{n}^{2}\right| \leq\left\|\chi_{1}-\chi_{2}\right\|_{L^{\infty}} .
$$

Proof. Note that for $i=1,2, H_{i}$ is a self-adjoint operator. Moreover, since $\chi_{i}$ is bounded from below, it is readily seen that $H_{i}$ is bounded from below. More specifically we have

$$
\left\langle H_{i} f, f\right\rangle \geq\left(\lambda_{1}+\min _{K} \chi_{i}\right)\|f\|_{L^{2}}^{2}
$$

where $\lambda_{1}$ is the smallest (positive) eigenvalue of $-\Delta$ on $K$. Therefore, by [12, Theorem XIII.2], it follows that the eigenvalues of the operators are given by

$$
\nu_{n}^{i}=\sup _{\phi_{1}, \phi_{2}, \cdots, \phi_{n-1}} \inf _{\psi \in\left[\phi_{1}, \cdots, \phi_{n-1}\right]^{\perp} ;\|\psi\|_{L^{2}}=1}\left\langle H_{i} \psi, \psi\right\rangle .
$$

Moreover, we have the following estimate:

$$
\begin{aligned}
\left\langle H_{1} \psi, \psi\right\rangle & =\left\langle H_{2} \psi, \psi\right\rangle+\left\langle\left(\chi_{1}-\chi_{2}\right) \psi, \psi\right\rangle \\
& \leq\left\langle H_{2} \psi, \psi\right\rangle+\left\|\chi_{1}-\chi_{2}\right\|_{L^{\infty}}\|\psi\|_{L^{2}}^{2} .
\end{aligned}
$$

Using (41) together with (3) completes the proof.

\section{SCHRÖDINGER OPERATORS WITH STEP FUNCTION POTENTIAL}

Let $N>1$ be an integer and consider a partition of the Sierpiński gasket into

$$
K=\bigcup_{|\omega|=N} F_{\omega} K,
$$

where for each word $\omega$ of length $N, F_{\omega} K$ is an $N$ cell. Note that there are exactly $3^{N}$ such cells, each of which has a measure $3^{-N}$. Consider a real-valued function associated to this partition: $\chi_{N}=\sum_{j=1}^{3^{N}} a_{j} \chi_{C_{j}}$, where the constants $a_{j} \in \mathbb{R}$ are not necessarily all distinct, and where $C_{j}=F_{\omega} K$ for some word $\omega$ of length $N$.

We wish in this section to study the asymptotics of the spectrum of the Schrödinger operator $H_{N}=-\Delta+\chi_{N}$.

We adopt the notation of [4, and let $\lambda_{k}:=5^{k-1} \lambda_{n}^{(6)}$ be one of the eigenvalues corresponding to the 6 -series as described above. Note that we ignore the second index $n$, as it is irrelevant for our investigation. Additionally, because we are looking at the limiting behavior of the spectrum of $H_{N}$ as $k \rightarrow \infty$, we may assume without loss of generality that $k>>N$. Let $E_{k}$ be the eigenspace associated to $\lambda_{k}$, and set $E_{k}=\operatorname{span}\left\{u_{l}^{k}, l=1,2, \ldots, d_{k}\right\}$ where $d_{k}=\operatorname{dim} E_{k}=\frac{3^{k}-3}{2}$ is the multiplicity of $\lambda_{k}$. Denote by $\tilde{\Lambda}_{N}^{k}$ the portion of the spectrum of $H_{N}$ lying in the 
interval $\left[\lambda_{k}+\min \chi_{N}, \lambda_{k}+\max \chi_{N}\right]$, and denote the eigenvalues in $\tilde{\Lambda}_{N}^{k}$ by $\left\{\tilde{\lambda}_{l}^{k}\right\}$ (counting multiplicity). We claim that, for $k$ large enough, $\tilde{\Lambda}_{N}^{k}$ contains exactly $d_{k}$ eigenvalues. This follows from Lemma 1, since the separation between $\lambda_{k}$ and the next higher and lower eigenvalues of $\Delta$ grows exponentially in $k$, and so eventually exceeds $\left\|\chi_{N}\right\|_{L^{\infty}}$.

Because the $\lambda_{k}$-eigenfunctions are localized eigenfunctions, we may choose $u_{l}^{k}$ so that the first $\tilde{d}_{k}$ of them are entirely supported in a single $N$ cell, where $\tilde{d}_{k}=3^{N} m_{N}^{k}$, with $m_{N}^{k}$ representing the multiplicity of eigenfunctions supported in a given $N$ cell. Denote by $\tilde{E}_{k}$ the span of these localized eigenfunctions. It follows from the description of the spectrum of $\Delta$ in 4 , that $m_{N}^{k}=\frac{3^{k-N}-3}{2}$. Each of the $m_{N}^{k}$ eigenfunctions $u_{l}^{k}$ supported in the cell $C_{j}$ is trivially seen to be an eigenfunction of $H_{N}$. Indeed, it follows from the form of the potential $\chi_{N}$ that $H_{N} u_{l}^{k}=$ $\left(\lambda_{k}+a_{j}\right) u_{l}^{k}$, for all $l=1,2, \ldots, m_{N}^{k}$. Thus, $\lambda_{j}^{k}=\lambda_{k}+a_{j}$ is an eigenvalue of $H_{N}$ with multiplicity of at least $m_{N}^{k}$. Note that these eigenvalues all belong to the interval $\left[\lambda_{k}+\min \chi_{N}, \lambda_{k}+\max \chi_{N}\right]$. So we have identified $\tilde{d}_{k}$ eigenvalues in $\tilde{\Lambda}_{N}^{k}$, and the remaining number of eigenvalues is $\alpha_{N}^{k}=d_{k}-\tilde{d}_{k}=\frac{3^{N+1}-3}{2}$, which is independent of $k$, so $\lim _{k \rightarrow \infty} \frac{\alpha_{N}^{k}}{d_{k}}=0$.

Define the characteristic measure of the subset $\tilde{\Lambda}_{N}$ of the spectrum of $H_{N}$ by

$$
\begin{aligned}
\phi_{k}^{N}(\lambda) & =\frac{1}{d_{k}} \sum_{l=1}^{d_{k}} \delta\left[\lambda-\left(\tilde{\lambda}_{l}^{k}-\lambda_{k}\right)\right] \\
& =\frac{m_{N}^{k}}{d_{k}} \sum_{j=1}^{3^{N}} \delta\left[\lambda-\left(\lambda_{j}^{k}-\lambda_{k}\right)\right]+\frac{1}{d_{k}} \sum_{l \in A_{N}^{k}} \delta\left[\lambda-\left(\tilde{\lambda}_{l}^{k}-\lambda_{k}\right)\right] \\
& =\frac{m_{N}^{k}}{d_{k}} \sum_{j=1}^{3^{N}} \delta\left(\lambda-a_{j}\right)+\frac{1}{d_{k}} \sum_{l \in A_{N}^{k}} \delta\left[\lambda-\left(\tilde{\lambda}_{l}^{k}-\lambda_{k}\right)\right],
\end{aligned}
$$

where $\# A_{N}^{k}=\alpha_{N}^{k}$. Observe that the factor $m_{N}^{k}$ in the definition of $\phi_{k}^{N}$ is due to the fact that the eigenvalue $\lambda_{j}^{k}$ has multiplicity $m_{N}^{k}$. We prove below that the measures $\phi_{k}^{N}$ converge to a measure $\phi_{0}^{N}$, defined to be the pullback of $\mu$ under $\chi_{N}$. More specifically, $\phi_{0}^{N}$ is defined by

$$
\phi_{0}^{N}(\lambda)=\sum_{j=1}^{3^{N}} \mu\left(C_{j}\right) \delta\left(\lambda-a_{j}\right)=3^{-N} \sum_{j=1}^{3^{N}} \delta\left(\lambda-a_{j}\right),
$$

or equivalently, for any continuous bounded function $f$,

$$
\left\langle\phi_{0}^{N}, f\right\rangle=\int_{K} f\left(\chi_{N}(x)\right) d \mu(x) .
$$

Lemma 2. $\phi_{k}^{N} \rightarrow \phi_{0}^{N}$ weakly as $k \rightarrow \infty$; in other words, for any continuous and bounded function $f \in \mathcal{C}_{b}(\mathbb{R})$,

$$
\lim _{k \rightarrow \infty}\left\langle\phi_{k}^{N}, f\right\rangle=\left\langle\phi_{0}^{N}, f\right\rangle .
$$


Proof. Let $f \in \mathcal{C}_{b}(\mathbb{R})$,

$$
\begin{aligned}
\left|\left\langle\phi_{k}^{N}-\phi_{0}^{N}, f\right\rangle\right| & \leq \sum_{j=1}^{3^{N}}\left|\left(\frac{m_{N}^{k}}{d_{k}}-3^{-N}\right) f\left(a_{j}\right)\right|+\frac{1}{d_{k}} \sum_{l \in A_{N}^{k}}\left|f\left(\tilde{\lambda}_{l}^{k}-\lambda_{k}\right)\right| \\
& \leq\|f\|_{L^{\infty}} \frac{3^{N+1}-3}{3^{k}-3}+\|f\|_{L^{\infty}} \frac{\alpha_{N}^{k}}{d_{k}} \\
& =2\|f\|_{L^{\infty}} \frac{3^{N+1}-3}{3^{k}-3} .
\end{aligned}
$$

Letting $k \rightarrow \infty$ in (8) concludes the proof of the lemma.

\section{SCHRÖDINGER OPERATOR WITH CONTINUOUS POTENTIAL}

In this section we would like to investigate the asymptotics of the eigenvalues of the Schrödinger operator $H=-\Delta+\chi$. For each $N \geq 1$ construct a partition of $K$ as in (5). Any continuous function $\chi \in \mathcal{C}(K)$ can be approximated uniformly by a sequence of simple functions $\chi_{N}=\sum_{j=1}^{3^{N}} a_{j} \chi_{C_{j}}$. Then Lemma 1 says that the spectrum of $H$ is approximated by the spectrum of $H_{N}$. In particular, if $\tilde{\Lambda}^{k}$ denotes the portion of the spectrum of $H$ contained in the interval $\left[\lambda_{k}+\min \chi\right.$, $\left.\lambda_{k}+\max \chi\right]$, then $\tilde{\Lambda}^{k}$ is approximated by $\tilde{\Lambda}_{N}^{k}$ (we can choose the approximation so that $\min \chi \leq \min \chi_{N}$ and $\max \chi_{N} \leq \max \chi$ ). As before, for $k$ large enough, $\tilde{\Lambda}^{k}$ contains $d_{k}$ eigenvalues which we will denote $\left\{\nu_{l}^{k}\right\}_{l=1}^{d_{k}}$. We call this the $\lambda_{k}$ cluster of the eigenvalues of $H$.

Define the characteristic measure of the $\lambda_{k}$ cluster of $H$ by

$$
\psi_{k}=\frac{1}{d_{k}} \sum_{l=1}^{d_{k}} \delta\left[\lambda-\left(\nu_{l}^{k}-\lambda_{k}\right)\right] .
$$

We show below that these measures converge to the pullback of the measure $\mu$ on $S G$ under $\chi$ defined by

$$
\left\langle\psi_{0}, f\right\rangle=\int_{K} f(\chi(x)) d \mu(x)
$$

for all test functions $f$.

As mentioned in Section 2, for each $k$ large enough, most of the eigenvalues of $H_{N}$ are just $\lambda_{j}^{k}=\lambda_{k}+a_{j}$, which are just shifts of $\lambda_{k}$ by $-a_{j}$. Moreover, according to Lemma 1, for all $k$ and $l$

$$
\left|\nu_{l}^{k}-\tilde{\lambda}_{l}^{k}\right| \leq\left\|\chi-\chi_{N}\right\|_{L^{\infty}} .
$$

Theorem 1. $\psi_{k} \rightarrow \psi_{0}$ weakly as $k \rightarrow \infty$, i.e., for all continuous bounded functions $f \in \mathcal{C}_{b}(\mathbb{R})$,

$$
\lim _{k \rightarrow \infty}\left\langle\psi_{k}, f\right\rangle=\left\langle\psi_{0}, f\right\rangle .
$$

Proof. Given any $f \in \mathcal{C}_{b}(\mathbb{R})$, let $\epsilon, \eta>0$.

$$
\begin{aligned}
\left\langle\psi_{k}-\psi_{0}, f\right\rangle & =\left\langle\psi_{k}-\phi_{k}^{N}, f\right\rangle+\left\langle\phi_{k}^{N}-\phi_{0}^{N}, f\right\rangle+\left\langle\phi_{0}^{N}-\psi_{0}, f\right\rangle \\
& =I+I I+I I I
\end{aligned}
$$

where $\phi_{k}^{N}$ and $\phi_{0}^{N}$ were previously defined in Section 2 .

Choose $N_{0} \geq 1$ such that for all $N \geq N_{0}$,

$$
\left\|\chi-\chi_{N}\right\|_{L^{\infty}} \leq \eta .
$$


Now let $N \geq N_{0}$ be fixed but arbitrary. Notice that $f$ is uniformly continuous on the compact set

$$
\chi(K) \cup \chi_{N}(K)=\chi(K) \bigcup_{j=1}^{3^{N}}\left\{a_{j}\right\} .
$$

Therefore, there exists $\alpha>0$ such that for all $z_{1}, z_{2} \in \chi(K) \cup \chi_{N}(K)$ with $\left|z_{1}-z_{2}\right| \leq$ $\alpha$, we have

$$
\left|f\left(z_{1}\right)-f\left(z_{2}\right)\right| \leq \epsilon / 3
$$

By choosing $\eta=\alpha$ in (11), it follows that

$$
|I I I| \leq \int_{K}\left|f(\chi(x))-f\left(\chi_{N}(x)\right)\right| d x \leq \epsilon / 3 .
$$

Moreover, by Lemma 2, it is readily seen that

$$
|I I| \leq 2\|f\|_{L^{\infty}}\left(\frac{3^{N+1}-3}{3^{k}-3}\right) .
$$

Consequently, we can choose $l_{0}>>N$ such that for all $k \geq l_{0}$ we have

$$
|I I| \leq \epsilon / 3 \text {. }
$$

Note that $\nu_{l}^{k}$ and $\tilde{\lambda}_{l}^{k}$ belong to the interval $\left[\lambda_{k}+\min \chi, \lambda_{k}+\max \chi\right]$, and $f$ is uniformly continuous on this interval and on $\chi(K) \cup \chi_{N}(K)$. Using Lemma 1 and the observations made above, one can choose $0<\alpha^{\prime} \leq \alpha$ such that for all $a, b$ in the above set, with $|a-b| \leq \alpha^{\prime}$, we have $|f(a)-f(b)| \leq \epsilon / 3$. Therefore,

$$
\begin{aligned}
|I| & \leq \frac{1}{d_{k}} \sum_{l=1}^{d_{k}}\left|f\left(\nu_{l}^{k}\right)-f\left(\tilde{\lambda}_{l}^{k}\right)\right| \\
& \leq \frac{1}{d_{k}} \sum_{l=1}^{d_{k}} \epsilon / 3 \\
& =\epsilon / 3 .
\end{aligned}
$$

Combining (12), (13) and (14) we conclude the proof of the theorem.

Remark 1. Similar results can be proved for the Neumann spectrum. Since the proof is quite similar to the one above, we omit it.

One could do the same analysis starting from the 5 -series eigenvalues of $-\Delta$. However, in this case the analysis is a little different. In particular, the proof of Theorem 1 relies on the existence of large gaps between the (6-series) eigenvalues of $-\Delta$. But the key argument when dealing with the 5 -series eigenvalues is that while other eigenvalues of $H$ can be very close, it is true that for a given cluster, the number of (other) eigenvalues, counting multiplicity, that are nearby is bounded by a constant that only depends on the size of the neighborhood.

Our results are also likely to hold on all pcf fractals with localized eigenfunctions as described in 2 .

\section{REFERENCES}

1. M. T. Barlow, Diffusion on Fractals, in: Lectures Notes in Mathematics, Vol. 1690, Springer, Berlin, 1998. MR 1668115 (2000a:60148)

2. M. T. Barlow and J. Kigami, Localized eigenfunctions of the Laplacian on p.c.f. self-similar sets, J. London Math. Soc., 56 (1997), no. 2, 320-332. MR1489140 (99b:35162) 
3. K. Coletta, K. Dias and R. S. Strichartz, Numerical analysis on the Sierpinski gasket, with applications to Schrödinger equations, wave equation, and Gibbs' phenomenon, Fractals, 12 (2004), no. 4, 413-449. MR2109985 (2005k:65245)

4. K. Dalrymple, R. S. Strichartz, and J. P. Vinson, Fractal differential equations on the Sierpinski gasket, J. Fourier Anal. Appl., 5 (1999), no. 2/3, 203-284. MR1683211(2000k:31016)

5. M. Fukushima and T. Shima, On a spectral analysis for the Sierpinski gasket, Potential Anal., 1 (1992), 1-35. MR1245223 (95b:31009)

6. V. Guillemin, Some spectral results on rank one symmetric spaces, Advances in Math., 28 (1978), 129-137. MR0494331(58:13228a)

7. V. Guillemin, An Addendum to: Some spectral results on rank one symmetric spaces, Advances in Math., 28 (1978), 138-147. MR0494332 (58:13228b)

8. V. Guillemin, Some spectral results for the Laplace operator with potential on the $n$-sphere, Advances in Math., 27 (1978), 273-286. MR0478245 (57:17730)

9. J. Kigami, Harmonic calculus on p.c.f. self-similar sets, Trans. Amer. Math. Soc., 335 (1993), 721-755. MR1076617 (93d:39008)

10. J. Kigami, "Analysis on Fractals," Cambridge University Press, New York, 2001. MR1840042 (2002c:28015)

11. R. Rammal and G. Toulouse, Random walks on fractal structures and percoloration clusters, J. Physique Lett., 44 (1983), L13-L22.

12. M. Reed, and B. Simon, "Methods of modern mathematical physics, IV, Analysis of Operators," Academic Press, New York-London, 1978. MR0493421 (58:12429c)

13. R. S. Strichartz, Analysis on fractals, Notices Amer. Math. Soc., 46 (1999), 1199-1208. MR.1715511 (2000i:58035)

14. R. S. Strichartz, "Differential Equations on Fractals: a Tutorial," Princeton University Press, Princeton, NJ, 2006. MR 2246975

15. A. Teplyaev, Spectral Analysis on Infinite Sierpiński Gaskets, J. Funct. Anal., 159 (1998), 537-567. MR1658094 (99j:35153)

16. A. Weinstein, Asymptotics of eigenvalue clusters for the Laplacian plus a potential, Duke Math. J., 44 (1977), no. 4, 883-892. MR0482878 (58:2919)

Department of Mathematics, Malott Hall, Cornell University, Ithaca, New York $14853-4201$

Current address: Department of Mathematics, University of Maryland, College Park, Maryland 20742-4015

E-mail address: kasso@math.umd.edu

Department of Mathematics, Malott Hall, Cornell University, Ithaca, New York 14853-4201

E-mail address: str@math.cornell.edu 